\title{
ROLE OF DIASTOLIC ARTERIAL PRESSURE IN MICROSTRUCTURAL CHANGES OF THE BRAIN IN PATIENTS WITH HYPERTENSION-RELATED CEREBRAL SMALL VESSEL DISEASE.
}

\author{
Kamila V. Shamtieva, Larisa A. Dobrynina, Elena I. Kremneva, Marina V. Krotenkova, Lyudmila A. Kalashnikova, \\ Elena V. Gnedovskaya, Aleksandr B. Berdalin.
}

Introduction. The usage of modern antihypertensive drugs improved the course of arterial hypertension (AH), but did not lead to the expected reduction in the incidence of small vessel disease (SVD) and its complications. This fact encourages further investigation of the mechanisms of hypertension-related brain damage.

Objective: Evaluation of the relation between daily profile of blood pressure (BP) and microstructure changes of the brain in patients with SVD and AH.

Material and methods. The study included 64 patients (mean age $59.4 \pm 5.4$ years, $38(59,4 \%)$ female) with SVD and AH. All patients received antihypertensive drugs. Ambulatory blood pressure monitoring (ABPM) and diffusion MRI with region of interest analysis of diffusion coefficient according to different maps for all participants. Regions of interest were taken separately in the juxtacortical, deep and periventricular zones in the hyperintensity and normal-appeared white matter of anterior and posterior frontal lobes, temporal-parietal area of the left hemisphere (fig. 1); anterior, middle, posterior cingulate gyrus; corpus callosum; amygdala and hippocampus. The relation between parameters was estimated using multivariate linear regression.

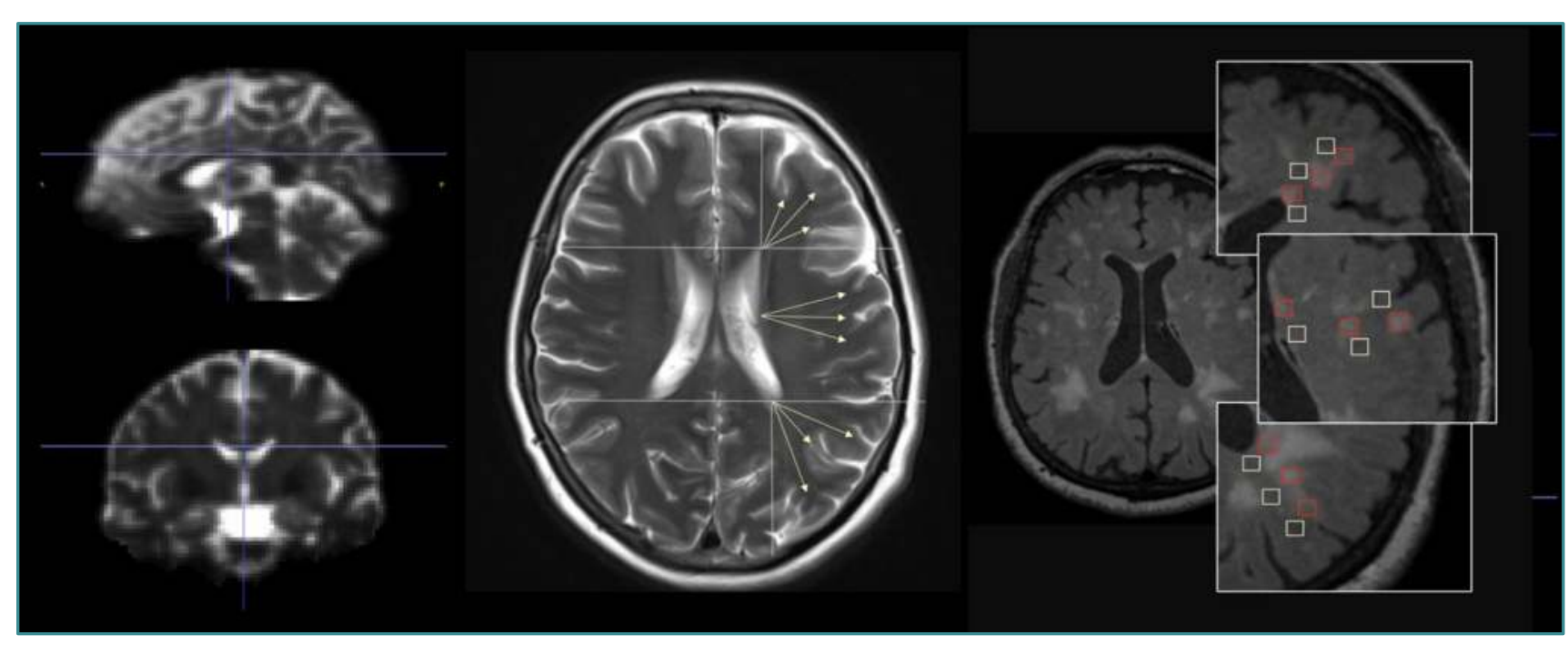

Figure 1. Estimation of diffusion parameters: (fractional anisotropy - FA, mean diffusivity - MD, radial diffusivity - RD, axial diffusivity - AD) in regions of interest.

Selection of the level of the brain slice and regions of interest in the white matter of the left hemisphere separately in the juxtacortical, deep and periventricular zones [1]:

red squares - white matter hyperintensity,

white squares - normal-appearing white matter.
Results. A statistically significant relation was found between daily profile of BP according to ABPM and microstructure abnormalities of the juxtacortical white matter hyperintensity (jWMH) of anterior and posterior frontal lobes according to MRI data. An increase in diastolic blood pressure (DBP) and its variability were of predominant importance in increasing in mean, radial and axial diffusivity.

Conclusion. The revealed relations evidence to importance of the increase and variability of DBP to brain damage in patients with SVD and AH. Microstructural abnormalities seems to correspond to an increase in the diffusion of free water and damage to myelin suggesting an increase in permeability of the blood-brain barrier and the development of vasogenic edema in areas of $\mathrm{jWMH}$ in patients with SVD and AH.

Table 1. Relationship between blood pressure (BP) profile according to ambulatory BP monitoring and DTI data (multiple linear regression analysis, models with $R^{2}>0,6, p<0,05$ )

\begin{tabular}{|c|c|c|}
\hline Model: variables & $\beta$ & $\mathbf{p}$ \\
\hline \multicolumn{3}{|l|}{ MD in jWMH anterior frontal lobes $\left(R^{2}=0,992\right)$ : } \\
\hline variability of diastolic BP during day & $5,30 * 10^{-5}$ & 0,001 \\
\hline variability of diastolic BP during night & $4,12^{*} 10^{-5}$ & 0,003 \\
\hline variability of systolic BP during night & $-2,08 * 10^{-5}$ & 0,019 \\
\hline constant & 0,001 & $<0,001$ \\
\hline \multicolumn{3}{|l|}{ RD in jWMH anterior frontal lobes $\left(R^{2}=1\right)$ : } \\
\hline variability of diastolic BP during day & $4,81 * 10^{-5}$ & $<0,001$ \\
\hline variability of diastolic BP during night & $2,69 * 10^{-5}$ & $<0,001$ \\
\hline hypertension load time index of systolic BP during day & $-1,75^{*} 10^{-6}$ & 0,001 \\
\hline maximum systolic BP during day & $-4,05^{*} 10^{-7}$ & 0,014 \\
\hline constant & 0,001 & $<0,001$ \\
\hline \multicolumn{3}{|l|}{ AD in jWMH anterior frontal lobes $\left(R^{2}=0,655\right)$ : } \\
\hline variability of diastolic BP during day & $4,7 * 10^{-5}$ & 0,027 \\
\hline constant & 0,001 & $<0,001$ \\
\hline \multicolumn{3}{|l|}{ MD in jWMH posterior frontal lobes $\left(R^{2}=0,603\right)$ : } \\
\hline variability of diastolic BP during night & $-3,9 * 10^{-6}$ & 0,003 \\
\hline hypertension load area index of systolic BP during day & $1,7 * 10^{-6}$ & 0,002 \\
\hline hypertension load time index of diastolic BP during day & $-3,8 * 10^{-6}$ & 0,025 \\
\hline constant & 0,001 & $<0,001$ \\
\hline
\end{tabular}

Note. $\beta$, regression coefficient for each variable.

The predicted value of the dependent variable can be calculated by the multiple linear regression equation: $y=$ constant $+\beta_{1} x_{1}+\beta_{2} x_{2}+\beta_{3} x_{3}+\ldots+\beta_{p} x$. 